\title{
Determination of Local Structure in Meteoritic Nanoscale Porous Carbon to Explain Entrapped Noble Gases
}

\author{
Rhonda M. Stroud ${ }^{1}$, Matthew F. Chisholm² Sachiko Amari $^{3}$, Jun-ichi Matsuda ${ }^{4}$ \\ ${ }^{1}$ Materials Science and Technology Division, Naval Research Laboratory, Washington, DC 20375 \\ ${ }^{2}$ Materials Science and Technology Division, Oak Ridge National Laboratory, Oak Ridge, TN \\ 37831-6069, USA \\ ${ }^{3}$ Department of Physics, Washington University, St. Louis, MO 63130, USA \\ ${ }^{4}$ Department of Earth and Space Sciences, Osaka University, Osaka, 560-0043, Japan
}

Nanoscale carbonaceous phases found in meteorites originated in a variety of astrophysical environments: as condensed dust around stars, in the interstellar medium, and in the early solar system. Known phases include graphite, $\mathrm{SiC}$ and nanodiamond, acid-resistant amorphous carbon (a.k.a "glassy"carbon) and macromolecular organic carbon. The noble gases trapped in the different carbonaceous phases reflect the original formation environments, and subsequent alteration histories. One noble gas component, "Q", is present in all primitive meteorites, but a definitive identification of the carrier phase(s) of "Q" remains elusive [1]. The trapped "Q" gases can be concentrated by acid dissolution of the host meteorite and density separation of the insoluble residue. Prior TEM studies of "Q"-rich residues from the Allende meteorite indicate the presence of curled graphene, however the residues contained multiple carbonaceous phases, so a definitive identification of the carrier could not be made [2]. We seek to identify the "Q" carrier(s) through electron microscopy of the "Q"-rich separates from the Saratov meteorite, which have high trapped gas contents, and but no graphite, $\mathrm{SiC}$ or nanodiamond [3].

Residues of the Saratov meteorite were prepared by $\mathrm{HF}-\mathrm{HCl}$ acid treatment and repeated colloidal separation [4]. For electron microscopy studies, the colloidal (AI) and non-colloidal (AJ) fractions were pipetted onto lacey carbon films on $\mathrm{Cu}$ TEM grids. Conventional bright-field imaging, highresolution imaging and selected area diffraction studies were performed with the JEOL 2200FS TEM at NRL. Aberration-corrected STEM imaging and electron energy loss spectroscopy (EELS) studies were carried out with the Nion UltraSTEM 100 at ORNL, operated at $60 \mathrm{kV}$ with a nominal probe size of $120 \mathrm{pm}$.

Bright-field TEM imaging and selected area diffraction (SAD) of the AJ and AI samples reveals only two phases: porous amorphous carbon and chromite (Fig. 1). We discount the possibility that the chromite grains contain significant " $Q$ " gases because the chromites survive oxidation treatments that remove the "Q" gases and carbon. The SAD patterns of the porous carbon indicate that it consists of randomly-oriented nanoscale domains of graphene.

Aberration-corrected annular dark-field (ADF) STEM imaging (Fig. 2) shows local variation in the aromatic domain size, flatness, edge termination and packing. The edges of some domains appear to be curled, connecting back on themselves or adjacent domains. This is consistent with calculations that indicate that a tubular edge termination is the most stable structure for non-H-terminated graphene nanoribbons [5]. In addition, the ADF images show that impurity atoms are primarily restricted to the edges of the graphene domains. 
Our results indicate that the carrier of "Q" is one or more of the morphologies contained in the porous amorphous carbon. It is known from the release temperatures of the " $Q$ " gases that the gases are physical trapped or bonded into the carbon network. Likely trapping sites are in the curled edges of the graphene domains. However, a definitive identification of the specific carrier morphology or morphologies, will require direct imaging, or spectroscopic detection of the trapped gas atoms. This is experimentally challenging, due to the low abundance of gas atoms, e.g., $1 \mathrm{Xe}$ per $10^{9} \mathrm{C}$. At this concentration, the residue volume sampled with selected area diffraction $\left(0.1 \mathrm{um}^{3}\right)$ contains on average $\sim 1 \mathrm{Xe}$ atom. Thus, on average the carrier morphology is represented in each diffraction pattern, although the majority of material analyzed is not associated with the "Q" gases. We obtained several diffraction patterns from each residue, and did not detect any phase other than nanoscale graphene and chromite, but a rare nanocrystalline carbon component could be obscured by the chromite diffraction spots. Additional studies are planned, to test the feasibility of automated analysis of ADF images to identify any trapped Xe.

\section{References:}

[1] Wieler, R. et al. in Meteorites and the Early Solar System II (University of Arizona Press, Tuscon) pp. 499-521 (2006).

[2] Matsuda, J. et al. Geochimica et Cosmochemica Acta, 74, 5398-5409 (2010).

[3] Matsuda, J. et al. Meteor. \& Planet. Sci. 45 361-372 (2010).

[4] Amari, S. and Matsuda, J. 43rd Lunar \& Planetary Science Conference Abstract \#1051 (2012).

[5] Ivanovskaya, V. V. et al. Phys. Rev. Lett. 107, 065502 (2011).

[6] This work was supported in part by the DOE Office of Basic Energy Sciences, Materials Sciences and Engineering Division (M.F.C.), and the NASA Cosmochemistry program.

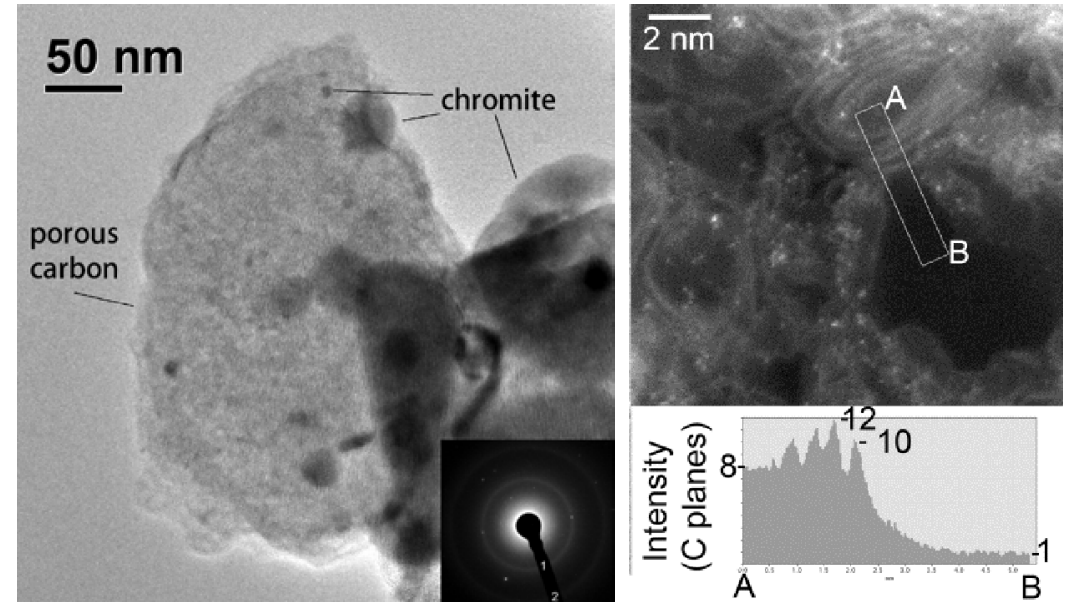

Fig. 1.(left) Bright field TEM of "Q" gas- bearing residues shwoing carbon and chromite phases. Selected area diffraction (left inset) reveals (10 $\overline{1} 0)$ and $(11 \overline{2} 0)$ graphene order in the porous carbon. (right) Aberrationcorrected annular dark-field image of the porous carbon, with intesity profile nromalized to carbon planes thickness. The edges of stacked graphene domains show extra planes, indicative of curled edges.

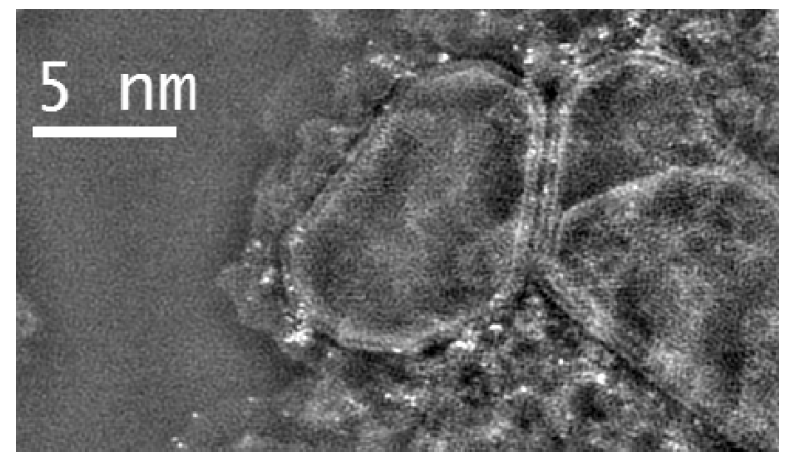

Fig. 2. FFT-filtered aberration-corrected annular darkfield image of the porous carbon, showing local variation in the microstrcuture of the graphene domains. Gas entrapment sites are most likley at the edges of the domains. 